\section{Validez de contenido de la Escala de Autoeficacia} Colectiva Docente
Abril 2021, Vol. 13, №1, 59-72

revistas.unc.edu.ar/inde x.php/racc

Sánchez-Rosas, Javier*, a ; Dyzenchauz, Malena ${ }^{a}$; Domínguez-Lara, Sergio ${ }^{\text {b }}$

Artículo Original

Resumen

La autoeficacia colectiva docente, entendida como las percepciones compartidas de los docentes de una escuela de que, en conjunto, podrán organizar y ejecutar las acciones necesarias para ejercer un efecto positivo en los estudiantes, se relacionó con diferentes variables asociadas a la educación. A pesar de la importancia que adquirió en los últimos años, los instrumentos creados para medirla presentan limitaciones. Por eso, el presente trabajo se propone desarrollar un instrumento para evaluarla y obtener evidencias de validez de contenido. Para ello, se redactaron los ítems junto con docentes e investigadores, de acuerdo a las seis dimensiones de la autoeficacia docente propuestas por Bandura (2006). Luego se elaboró una prueba de juicio de expertos con una muestra de docentes y otra con una muestra de investigadores. Los ítems demostraron ser válidos en cuanto a su pertinencia y claridad, aunque se encontraron diferencias significativas entre las valoraciones de ambas muestras.

Palabras clave:

autoeficacia, psicometría, validez de contenido, docencia
Abstract

Content validity of the Collective Teacher Selfefficacy Scale. Collective teacher self-efficacy, understood as the shared perceptions of the teachers of a school that, together, they will be able to organize and execute the necessary actions to produce a positive effect on the students, was related to different variables associated to education. Despite the importance it has acquired in recent years, the instruments created to measure it have limitations. For this reason, the present work sets out to develop an instrument to evaluate it and obtain evidence of content validity. To do this, the items were written together with teachers and researchers, according to the six dimensions of teacher self-efficacy proposed by Bandura (2006). Then, an expert judgment test was prepared with a sample of teachers and another one with a sample of researchers. The items proved to be valid in terms of their relevance and clarity, although significant differences were found between the evaluations of both samples.

Keywords:

self-efficacy, psychometrics, content validity, teaching.

Recibido el 9 de abril de 2020; Aceptado el 20 de junio de 2020

Editaron este artículo: Raquel Peltzeer, Paula Abate, Nadia Justel y María Victoria Ortiz

\section{Introducción}

Los debates actuales coinciden en señalar que las políticas dirigidas a docentes son un factor determinante de la calidad de la enseñanza y de los aprendizajes de un país (Centro de Implementación de Políticas Públicas para la Equidad y el Crecimiento [CIPPEC], 2017). Sin embargo, se observa un notable malestar entre quienes ejercen la profesión (Cuenca et al., 2005). Entre los indicadores de este malestar se podrían señalar, por un lado, las percepciones sobre la propia capacidad para ejercer una influencia positiva sobre los estudiantes y, por otro lado, la falta de iniciativa y/o recursos institucionales para promover la actuación de los docentes como un colectivo organizado. $\mathrm{O}$, unificando estos dos enfoques, las dificultades que encuentran los docentes para llevar a cabo un trabajo de equipo que resulte en una mayor eficacia percibida y efectiva.

En esta dirección, el presente trabajo se

\footnotetext{
a Universidad Nacional de Córdoba, Facultad de Psicología, Laboratorio de Evaluación Psicológica y Educativa (LEPE), Córdoba, Argentina

b Universidad Privada San Juan Bautista, Lima, Perú

`Enviar correspondencia a: Sánchez-Rosas, J. E-mail: jsanchezrosas@unc.edu.ar
}

Citar este artículo como: Sánchez-Rosas, J., Dyzenchauz, M. \& Domínguez-Lara, S. (2021). Validez de contenido de la Escala de Autoeficacia Colectiva Docente. Revista Argentina de Ciencias del Comportamiento, 13(1), 59-72 
propone estudiar el constructo de autoeficacia colectiva docente. Si bien existen algunas escalas diseñadas para su medición (Skaalvik, E. \& Skaalvik, 2016; Tschannen-Moran \& Barr, 2004), presentan algunas falencias que limitan la validez de su utilización. Con el fin de contar con un instrumento apropiado, se busca elaborar una escala para medir la autoeficacia colectiva docente (ACD) con evidencias de validez de contenido basadas en el juicio de experto de investigadores y docentes (Merino-Soto, 2016, citado en Domínguez-Lara, 2017).

\section{Las dimensiones de la Autoeficacia Docente según Bandura}

La ACD puede definirse como las percepciones compartidas de los docentes de una escuela de que, en conjunto, podrán organizar y ejecutar las acciones necesarias para ejercer un efecto positivo en los estudiantes. Es un "producto de la dinámica interactiva de los miembros del grupo" (Goddard, Hoy, \& Woolfolk Hoy, 2000, p. 482) y representa una percepción compartida de los docentes sobre el funcionamiento de la escuela (Jean Eells, 2011).

Bandura (2006) propuso seis dimensiones para medir autoeficacia docente: autoeficacia para influenciar la toma de decisiones, instruccional, disciplinar, para conseguir el involucramiento parental, para conseguir el involucramiento comunitario y para crear un clima escolar positivo.

Si bien no define estas dimensiones ni justifica su selección, existe una amplia literatura que destaca su importancia. Por ejemplo, la influencia en la toma de decisiones se relacionó con el desempeño y la satisfacción laboral docente (Wagner, 1994), y con la productividad e innovación escolar (Somech, 2010). Las estrategias instruccionales desplegadas por los docentes se relacionaron positivamente con compromiso y motivación docente (Rodríguez, Núñez, Valle, Blas, \& Rosario, 2009), y con disfrute, valor de la tarea, atención y autoeficacia de los estudiantes (Esquivel \& Sánchez-Rosas, 2018; Sánchez-Rosas, Takaya, \& Molinari, 2016a, 2016b), y negativamente con el aburrimiento de los estudiantes (Sánchez-Rosas \& Esquivel, 2016). La disciplina estudiantil se relacionó con el desempeño académico (Gregory, Skiba, \& Noguera, 2010) y el clima escolar (Koth, Bradshaw, \& Leaf, 2008). El involucramiento parental mostró relaciones con el logro académico
(Kim \& Hill, 2015) y la motivación (Mendoza, 2012). El involucramiento de la comunidad se relacionó con el logro académico, la reducción del ausentismo y el mejoramiento de las políticas y programas escolares (Michael, Dittus, \& Epstein, 2007). Por último, el clima escolar se relacionó con la seguridad, las relaciones saludables y el compromiso en la enseñanza y en el aprendizaje (Thapa, Cohen, Guffey, \& Higgins-D'Alessandro, 2013).

\section{Medición de la autoeficacia colectiva docente}

El primer instrumento creado para medir ACD fue la Escala de Eficacia Colectiva Docente (Collective Teacher Efficacy Scale, CTES) de Goddard et al. (2000). Estos autores partieron de la Escala de Eficacia Docente de Gibson y Dembo (1984), diseñada para medir eficacia docente individual, y la adaptaron para medir la autoeficacia de manera colectiva reescribiendo las afirmaciones de individual a grupal. Luego de algunos ajustes y pruebas iniciales, administraron 21 ítems diseñados para evaluar dos dimensiones de la ACD: Competencia Grupal (CG) y al Análisis de la Tarea (AT) (Cooper, 2010). Posteriormente, Goddard (2002) creó una versión corta del instrumento (Collective Efficacy Scale, CES), que quedó configurada por 12 reactivos.

La Escala de Creencias de Eficacia Colectiva Docente de Tschannen-Moran y Barr (2004) es otro instrumento usado repetidamente en la investigación sobre ACD (Kirby \& DiPaola, 2011; Klassen, Tze, Betts, \& Gordon, 2011; Leithwood, Sun, \& Schumacker, 2019; Ma, Resendes, Scardamalia, \& Dobbie, 2019; Malinen \& Savolainen, 2016). Esta herramienta está basada en la Escala de Eficacia Docente del Estado de Ohio (Tschannen-Moran \& Woolfolk Hoy, 2001) y es un instrumento de 12 ítems que evalúa las percepciones colectivas de las prácticas de enseñanza y su capacidad de influir en la disciplina estudiantil.

Por último, Skaalvik, E. y Skaalvik (2007) diseñaron un instrumento unidimensional de siete ítems a partir de su escala de autoeficacia docente individual (Norwegian Teacher SelfEfficacy Scale; NTSES). El sujeto de las afirmaciones se modificó desde yo a nosotros o los docentes de esta escuela.

\section{Limitaciones de los instrumentos de ACD \\ Los instrumentos existentes presentan algunas limitaciones. En primer lugar, los ítems se}


redactaron reemplazando el yo por un nosotros. Esta ligera modificación es insuficiente para dar cuenta de una dimensión colectiva de la autoeficacia. En su guía para construir escalas de autoeficacia, Bandura postula que "los logros de un grupo son el producto no sólo del conocimiento y las habilidades de los diferentes miembros, sino también de la dinámica interactiva, coordinada y sinérgica de sus transacciones" (Bandura, 2006, p. 316). Por lo tanto, la Autoeficacia Colectiva no es simplemente la suma de las creencias de autoeficacia de cada miembro.

Además, algunos de los instrumentos presentan una falta de congruencia con la teoría, ya que muchos de los ítems refieren a características ambientales, por ejemplo, el abuso de drogas y alcohol en la comunidad hace que el aprendizaje sea difícil para los estudiantes de aquí (Klassen et al., 2011). Podría aventurarse que esto deriva de una confusión entre los conceptos de autoeficacia y expectativas de resultado, constructos relacionados pero diferentes.

Por otro lado, la redacción de los ítems de varios de los instrumentos mencionados se desvía de los parámetros sugeridos por Bandura (2006). Por ejemplo, en algunos de ellos (Skaalvik, E. \& Skaalvik, 2016; Tschannen-Moran \& Barr, 2004), los reactivos no son acciones o conductas sino preguntas que involucran la conducta. Incluso, en vez de preguntar por la confianza o seguridad para realizar un comportamiento, se pregunta por el grado de acuerdo o la veracidad de la pregunta y/o afirmación. Además, en estos dos instrumentos, el escalamiento tipo Likert va de 1 a 9 (Tschannen-Moran \& Barr, 2004) o de 1 a 5 (Skaalvik, E. \& Skaalvik, 2016), al contrario de la sugerencia de Bandura (2006) de utilizar un rango de 1 a 10.

Por último, el instrumento de Skaalvik, E. y Skaalvik (2007) ha sido escasamente utilizado en la investigación sobre ACD, probablemente porque las subescalas fueron propuestas a partir de una investigación sobre las expectativas de los docentes en Noruega. Así, estas dimensiones específicas podrían variar ampliamente según el contexto.

\section{Relevancia de la autoeficacia colectiva docente}

Por otro lado, si bien la investigación sobre este constructo tiene un desarrollo modesto, la ACD fue relacionada con diversas variables. Por ejemplo, demostró una relación positiva con factores referentes al cuerpo docente como el involucramiento en la toma de decisiones escolar (Goddard, 2002), el compromiso docente (Lee, Zhang, \& Yin, 2011), el consenso respecto de los objetivos (Kurz \& Knight, 2004), la capacidad de liderazgo (Olivier \& Hipp, 2019), la satisfacción laboral (Klassen, Usher, \& Bong, 2010), la persistencia para la superación de los obstáculos (Cantrell \& Callaway, 2008), la autoeficacia docente individual (Fives \& Looney, 2009) y el manejo y fortalecimiento de las relaciones sociales entre estudiantes (Lev \& Koslowsky, 2009). También fue relacionada directamente con variables de los estudiantes como el rendimiento académico (Jean Eells, 2011), y de la institución, como el liderazgo transformacional (Dussault, Payette, \& Leroux, 2008) y asociada con un clima escolar favorable (Lim \& Eo, 2014). De manera negativa, se relacionó con el estrés atribuido a la conducta estudiantil (Klassen, 2010), el nivel escolar, y el nivel/estrato socioeconómico (Adams \& Forsyth, 2006).

\section{El presente estudio}

Considerando las limitaciones de las escalas desarrolladas, la importancia del constructo y la necesidad de contar con un instrumento válido, este trabajo tuvo como objetivo desarrollar un instrumento que evalúe la autoeficacia colectiva docente y brindar las primeras evidencias de validez basadas en el contenido de sus ítems.

\section{Método}

\section{Participantes}

Se utilizaron dos muestras de participantes que evaluaron la pertinencia y claridad de cada ítem. La primera muestra estuvo compuesta por un grupo de 16 investigadores con conocimientos en la creación de instrumentos y en autoeficacia. La segunda muestra se compuso de un grupo de 10 docentes de primaria que ejercen su profesión en diversos puntos de la provincia de Córdoba, Argentina, con diversas trayectorias y años de experiencia.

\section{Instrumento}

Escala de ACD. Se trata de un instrumento de autoinforme que mide las creencias sobre las capacidades del equipo docente en el que se está inserto. Más específicamente, el instrumento se diseñó para evaluar, mediante 45 ítems, seis dimensiones de la ACD: autoeficacia para influir en la toma de decisiones (Podemos organizarnos 
para ser escuchados al tomar decisiones en la escuela, 8 ítems), autoeficacia para la enseñanza (Podemos compartir estrategias efectivas para mejorar el desempeño de los estudiantes con dificultades de aprendizaje, 8 ítems), autoeficacia para la convivencia (Podemos contribuir entre todos a que los estudiantes respeten las reglas escolares, 7 ítems), autoeficacia para el logro de la participación familiar (Podemos trabajar en conjunto para que las familias acompañen a sus hijos en las tareas escolares, 7 ítems), autoeficacia para el logro de la participación comunitaria (Podemos diseñar proyectos que involucren la participación de organizaciones comunitarias, 7 ítems) y autoeficacia para el logro de un clima escolar positivo (Podemos entablar relaciones positivas para hacer de la escuela un lugar confiable, 8 ítems).

La respuesta a los ítems se realizó en una escala tipo Likert para indicar el grado en que los docentes creían que podían llevar a cabo esas conductas desde (1) para nada seguro/a de que podemos hacerlo hasta (10) totalmente seguro/a de que podemos hacerlo. Bandura (2006) sugirió emplear respuestas de 1 a 10 puntos para lograr mayor sensibilidad y predictibilidad, ya que las personas suelen evadir las posiciones extremas, por lo que una escala de pocos puntos podría reducir el rango de respuestas a uno o dos puntos.

\section{Procedimiento}

Siguiendo a Montero y León (2007) el presente trabajo se enmarcó dentro del modelo empírico cuantitativo, de tipo instrumental. La estructura de la escala y la redacción de los ítems se basaron en la teoría clásica de los test, en la medida que se espera homogeneidad de los ítems. Asimismo, este procedimiento se basó en las recomendaciones de la literatura especializada detalladas a lo largo del manuscrito: explicación de un marco general donde se refieren las motivaciones para elaborar la escala, la definición de la variable medida, especificaciones con relación al test (número de ítems, formato, etc.), y construcción de los ítems (Muñiz \& FonsecaPedrero, 2019).

En primer lugar, se redactaron los ítems a partir de una estrategia racional, basados en el contenido teórico del constructo más que en el interés por discriminar entre grupos, el cual es el objetivo de las estrategias empíricas (Vera-
Villaroel \& Oblitas, 2005). Por tal motivo, algunos reactivos de la Escala de Autoeficacia Docente de Bandura (2006) fueron adaptados en términos de colectivo docente, mientras que otros se crearon con base en el modelo teórico de referencia y con la colaboración de docentes e investigadores, mediante entrevistas presenciales o virtuales. En todo el proceso se tuvieron en cuenta las seis dimensiones propuestas por Bandura (2006): autoeficacia para influir en la toma de decisiones, autoeficacia instruccional, autoeficacia disciplinaria, autoeficacia para el logro de la participación parental, autoeficacia para el logro de la participación comunitaria y autoeficacia para el logro de un clima escolar positivo. Sin embargo, se cambió la nominación de algunas de ellas por resultar desactualizada o poco familiar para el grupo objetivo. Específicamente, autoeficacia instruccional se reemplazó por autoeficacia para la enseñanza, autoeficacia disciplinaria se reemplazó por autoeficacia para la convivencia, y autoeficacia para el logro de la participación parental se reemplazó por autoeficacia para el logro de la participación familiar. Se elaboró una definición de cada una de las dimensiones y se utilizaron estas definiciones y ejemplos para guiar a los docentes e investigadores que contribuyeron en la redacción de los ítems.

Finalmente, se unificaron los ítems muy similares entre sí y se eliminaron los que no correspondían al constructo de ACD, quedando la escala conformada por 45 elementos: 8 para la dimensión autoeficacia para influir en la toma de decisiones, 8 para autoeficacia para la enseñanza, 7 para autoeficacia para la convivencia, 7 para autoeficacia para el logro de la participación familiar, 7 para autoeficacia para el logro de la participación comunitaria y 8 para autoeficacia para crear un clima positivo. Se consideró que con la cantidad de reactivos generados a modo de banco de ítems para cada dimensión, si llegara a eliminarse alguno luego del proceso de validación del contenido, quedarían suficientes ítems por dimensión para garantizar la representatividad del constructo.

Para redactar todas las afirmaciones se tuvieron en cuenta las pautas sugeridas por Bandura (2006). Es decir, se expresaron en términos de podemos hacer, indicando la percepción sobre la capacidad del conjunto. Para dar cuenta del carácter colectivo que se pretende medir, las conductas que expresan los ítems son 
comportamientos necesariamente colectivos, es decir, acciones coordinadas de los miembros del grupo.

Una vez redactados, los reactivos se sometieron a una prueba de juicio de expertos. Los protocolos administrados a investigadores y docentes informaron el objetivo de la investigación, población destinataria y modalidad de administración. El protocolo también incluyó una definición de cada dimensión, y los ítems correspondientes, así como un espacio para realizar observaciones sobre cada reactivo, y sobre el instrumento en general.

Así, un conjunto de investigadores evaluó su claridad y pertinencia, entendiendo que cada reactivo sería claro si no admitiera más de una interpretación posible, y sería pertinente si apuntara a obtener la información que se está buscando. Al mismo tiempo, un grupo de docentes completó el mismo protocolo, ya que es posible encontrar discrepancias en cuanto a la percepción de la claridad de los ítems entre la muestra objetivo del instrumento y los denominados expertos (Merino-Soto, 2016, citado en Domínguez-Lara, 2017).

A todos los participantes se les informaron los objetivos de la investigación. También se les informó que su participación era voluntaria y anónima y que sus datos personales se considerarían confidenciales y se utilizarían únicamente con fines investigativos.

\section{Análisis de datos}

Para aportar evidencias de validez de contenido se construyó un protocolo de evaluación para jueces, solicitando la valoración de los reactivos a un grupo de expertos académicos y docentes de educación básica. En ese sentido, fueron consultados en torno a la pertinencia y claridad de cada uno, utilizando una escala de cinco puntos desde mala (1) hasta excelente (5). Luego, con base en los comentarios, así como cuestiones teóricas del constructo y construcción de instrumentos, los ítems originales fueron modificados para lograr la mejor versión posible.

Para la cuantificación del grado de acuerdo entre los jueces se utilizó el coeficiente $\vee$ de Aiken y sus intervalos de confianza (IC; Merino-Soto \& Livia Segovia, 2009). La V de Aiken es un indicador que cuantifica el acuerdo entre jueces con relación a los ítems y su magnitud va de 0 a 1 . Por su parte, el IC representa el rango de posibles valores que podría asumir la $\mathrm{V}$ de Aiken bajo ciertas condiciones, considerando que los indicadores no son estáticos. En ese sentido, se esperaron valores mayores que .50 para el límite inferior de los IC (Domínguez-Lara, 2017), de manera que aún en el más desfavorable de los casos (límite inferior del IC) la $\mathrm{V}$ de Aiken sería mayor que .50. Por otro lado, para analizar el IC de la diferencia entre las $\vee$ de Aiken de expertos y docentes se utilizó un procedimiento recomendado por Merino-Soto (2018), y se consideró que existía una diferencia significativa entre las valoraciones de ambos grupos si el rango del IC no incluía el cero.

\section{Resultados}

En la Tabla 1 se detallan la media y el coeficiente $\mathrm{V}$ de Aiken de cada ítem, los intervalos de confianza y las diferencias de los mismos entre las puntuaciones de docentes y de investigadores. En general, los estadísticos aportaron evidencias favorables de la validez de los reactivos relacionadas con su claridad y su pertinencia para medir las dimensiones de autoeficacia colectiva docente consideradas.

Por un lado, todos los ítems alcanzaron el criterio establecido para el límite inferior de .50, excepto algunos cuyos valores resultaron marginalmente aceptables $(.47, .49, .42)$, tal es el caso de las valoraciones docentes. Por otro lado, las diferencias para los intervalos de confianza en las valoraciones de las dos muestras de jueces fueron significativas en varios casos, tanto para las valoraciones de pertinencia como claridad. Si bien estas diferencias fueron informativas de cierta discrepancia en las valoraciones entre investigadores y docentes, no justificaron la necesidad de eliminar ítems, ya que la media de las valoraciones, al igual que el coeficiente $V$ de Aiken, es en todos los casos muy positiva. De todos modos, las diferencias entre las valoraciones de ambas muestras y los comentarios realizados resultaron útiles para realizar modificaciones en varios de los reactivos, volviéndolos más breves y comprensibles.

La Tabla 1 expone las puntuaciones de los ítems redactados originalmente. El instrumento final con los ítems modificados de acuerdo a las valoraciones y comentarios del juicio de expertos se encuentra en el anexo. 
Sánchez-Rosas, J., Dyzenchauz, M. \& Domínguez-Lara, S. / RACC, 2021, Vol. 13, №1, 59-72

Tabla 1.

Valoraciones de docentes e investigadores sobre claridad y pertinencia de los items

\begin{tabular}{|c|c|c|c|c|c|c|c|}
\hline \multicolumn{3}{|c|}{ Docentes } & \multicolumn{4}{|c|}{ Investigadores } & \multirow{2}{*}{$\frac{\text { Diferencias }}{\text { IC VD - VI }}$} \\
\hline ATD & MD & VD & IC D 95\% & MI & VI & IC I $95 \%$ & \\
\hline$I 1 p$ & 3.50 & .62 & $.47-.75$ & 5.00 & 1.00 & $.94-1.00$ & $-.53,-.24$ \\
\hline I1c & 3.60 & .65 & $.49-.77$ & 3.94 & .73 & $.61-.82$ & $-.26, .09$ \\
\hline $12 p$ & 3.90 & .72 & $.57-.83$ & 5.00 & 1.00 & $.94-1.00$ & $-.43,-.15$ \\
\hline $12 c$ & 3.90 & .72 & $.57-.83$ & 4.38 & .84 & $.73-.91$ & $-.29, .04$ \\
\hline $13 p$ & 3.50 & .62 & $.47-.75$ & 4.60 & .90 & $.79-.95$ & $-.44,-.11$ \\
\hline $13 c$ & 3.70 & .67 & $.52-.79$ & 4.00 & .75 & $.62-.84$ & $-.25, .10$ \\
\hline $14 p$ & 3.70 & .67 & $.52-.79$ & 4.56 & .89 & $.79-.94$ & $-.38,-.06$ \\
\hline $14 \mathrm{c}$ & 3.90 & .72 & $.57-.83$ & 4.56 & .89 & $.79-.94$ & $-.33,-.02$ \\
\hline $15 p$ & 3.50 & .62 & $.47-.75$ & 5.00 & 1.00 & $.94-1.00$ & $-.53,-.24$ \\
\hline $15 \mathrm{c}$ & 3.70 & .67 & $.52-.79$ & 4.69 & .92 & $.83-.96$ & $-.41,-.10$ \\
\hline $16 p$ & 3.50 & .62 & $.47-.75$ & 4.94 & .98 & $.91-.99$ & $-.55,-.21$ \\
\hline $16 c$ & 3.50 & .62 & $.47-.75$ & 4.81 & .95 & $.87-.98$ & $-.48,-.18$ \\
\hline $17 p$ & 3.80 & .70 & $.54-.81$ & 4.81 & .95 & $.87-.98$ & $-.41,-.11$ \\
\hline $17 c$ & 3.80 & .70 & $.54-.81$ & 4.44 & .86 & $.75-.92$ & $-.33, .00$ \\
\hline $18 p$ & 3.70 & .67 & $.52-.79$ & 5.00 & 1.00 & $.94-1.00$ & $-.48,-.20$ \\
\hline $18 \mathrm{c}$ & 3.80 & .70 & $.54-.81$ & 4.63 & .90 & $.81-.95$ & $-.37,-.06$ \\
\hline $\mathrm{AE}$ & MD & VD & IC D 95\% & MI & VI & IC I 95\% & IC VD - VI \\
\hline $19 p$ & 4.30 & .82 & $.68-.91$ & 4.81 & .95 & $.87-.98$ & $-.27,-.01$ \\
\hline $19 c$ & 4.30 & .82 & $.68-.91$ & 4.44 & .86 & $.75-.92$ & $-.19, .10$ \\
\hline $110 p$ & 4.00 & .75 & $.59-.85$ & 4.94 & .98 & $.91-.99$ & $-.39,-.11$ \\
\hline $110 \mathrm{c}$ & 4.00 & .75 & $.59-.85$ & 4.63 & .90 & $.81-.95$ & $-.32,-.02$ \\
\hline $111 p$ & 4.20 & .80 & $.65-.89$ & 4.81 & .95 & $.87-.98$ & $-.30,-.03$ \\
\hline I11c & 4.20 & .80 & $.65-.89$ & 4.37 & .84 & $.73-.91$ & $-.21, .10$ \\
\hline $112 p$ & 4.00 & .75 & $.59-.85$ & 4.88 & .97 & $.89-.99$ & $-.38,-.09$ \\
\hline I12c & 4.10 & .77 & $.62-.87$ & 4.75 & .93 & $.85-.97$ & $-.32,-.03$ \\
\hline $113 p$ & 4.00 & .75 & $.59-.85$ & 4.75 & .93 & $.85-.97$ & $-.34,-.05$ \\
\hline $113 c$ & 3.90 & .72 & $.57-.83$ & 4.63 & .90 & $.81-.95$ & $-.34,-.04$ \\
\hline$I 14 p$ & 4.10 & .77 & $.62-.87$ & 4.94 & .98 & $.91-.99$ & $-.36,-.09$ \\
\hline I14c & 4.30 & .82 & $.68-.91$ & 4.44 & .86 & $.75-.92$ & $-.19, .10$ \\
\hline $115 p$ & 3.90 & .72 & $.57-.83$ & 4.79 & .94 & $.85-.98$ & $-.38,-.08$ \\
\hline $115 c$ & 4.10 & .77 & $.62-.87$ & 4.13 & .78 & $.66-.86$ & $-.18, .15$ \\
\hline $116 p$ & 4.10 & .77 & $.62-.87$ & 4.88 & .97 & $.89-.99$ & $-.35,-.07$ \\
\hline I16c & 4.10 & .77 & $.62-.87$ & 4.63 & .90 & $.81-.95$ & $-.29, .00$ \\
\hline ACE & MD & VD & IC D 95\% & MI & VI & IC I 95\% & IC VD - VI \\
\hline $117 p$ & 4.10 & .77 & $.62-.87$ & 4.88 & .97 & $.89-.99$ & $-.35, .02$ \\
\hline I17c & 3.90 & .72 & $.57-.83$ & 4.50 & .87 & $.77-.93$ & $-.31, .00$ \\
\hline I18p & 4.30 & .82 & $.68-.91$ & 4.94 & .98 & $.91-.99$ & $-.30,-.05$ \\
\hline I18c & 4.30 & .82 & $.68-.91$ & 4.56 & .89 & $.79-.94$ & $-.22, .06$ \\
\hline $119 p$ & 3.90 & .72 & $.57-.83$ & 5.00 & 1.00 & $.94-1.00$ & $-.43,-.15$ \\
\hline I19c & 3.90 & .72 & $.57-.83$ & 4.75 & .93 & $.85-.97$ & $-.37,-.07$ \\
\hline I20p & 3.70 & .67 & $.52-.79$ & 5.00 & 1.00 & $.94-1.00$ & $-.48,-.20$ \\
\hline $120 \mathrm{c}$ & 3.80 & .70 & $.54-.81$ & 4.81 & .95 & $.87-.98$ & $-.41,-.11$ \\
\hline
\end{tabular}


Sánchez-Rosas, J., Dyzenchauz, M. \& Domínguez-Lara, S. / RACC, 2021, Vol. 13, №1, 59-72

\begin{tabular}{|c|c|c|c|c|c|c|c|}
\hline I21p & 4.20 & .80 & $.65-.89$ & 4.87 & .96 & $.88-.99$ & $-.31,-.04$ \\
\hline I21c & 4.20 & .80 & $.65-.89$ & 3.69 & .67 & $.55-.77$ & $-.05, .28$ \\
\hline $122 p$ & 4.30 & .82 & $.68-.91$ & 4.87 & .96 & .88-.99 & $-.28,-.02$ \\
\hline I22c & 4.20 & .80 & $.65-.89$ & 4.38 & .84 & $.73-.91$ & $-.21, .10$ \\
\hline $123 p$ & 4.30 & .82 & $.68-.91$ & 4.81 & .95 & $.87-.98$ & $-.27,-.01$ \\
\hline $123 c$ & 4.30 & .82 & $.68-.91$ & 4.69 & .92 & $.83-.96$ & $-.25, .03$ \\
\hline APF & MD & VD & IC D 95\% & MI & VI & IC I 95\% & IC VD - VI \\
\hline $124 p$ & 3.60 & .65 & $.49-.77$ & 4.88 & .97 & $.89-.99$ & $-.48,-.18$ \\
\hline I24c & 3.70 & .67 & $.52-.79$ & 4.56 & .89 & $.79-.94$ & $-.38,-.06$ \\
\hline $125 p$ & 3.90 & .72 & $.57-.83$ & 4.88 & .97 & $.89-.99$ & $-.40,-.11$ \\
\hline $125 \mathrm{c}$ & 4.00 & .75 & $.59-.85$ & 4.69 & .92 & $.83-.96$ & $-.33,-.04$ \\
\hline $126 p$ & 4.10 & .77 & $.62-.87$ & 4.73 & .93 & $.83-.97$ & $-.32,-.02$ \\
\hline $126 c$ & 4.20 & .80 & $.65-.89$ & 4.69 & .92 & $.83-.96$ & $-.28, .01$ \\
\hline $127 p$ & 3.80 & .70 & $.54-.81$ & 4.94 & .98 & $.91-.99$ & $-.44,-.15$ \\
\hline I27c & 3.70 & .67 & $.52-.79$ & 4.75 & .93 & $.85-.97$ & $-.42,-.12$ \\
\hline $128 p$ & 4.30 & .82 & $.68-.91$ & 4.75 & .93 & $.85-.97$ & $-.26, .01$ \\
\hline $128 \mathrm{c}$ & 4.30 & .82 & $.68-.91$ & 4.25 & .81 & $.70-.88$ & $-.15, .15$ \\
\hline $129 p$ & 3.30 & .57 & $.42-.71$ & 4.63 & .90 & $.81-.95$ & $-.49,-.13$ \\
\hline $129 c$ & 3.50 & .62 & $.47-.75$ & 4.12 & .78 & $.66-.86$ & $-.33, .02$ \\
\hline $130 p$ & 4.00 & .75 & $.59-.85$ & 4.75 & .93 & $.85-.97$ & $-.34,-.05$ \\
\hline $130 c$ & 4.00 & .75 & $.59-.85$ & 4.44 & .86 & $.75-.92$ & $-.28, .04$ \\
\hline APC & MD & VD & IC D 95\% & MI & VI & IC I 95\% & IC VD - VI \\
\hline I31p & 4.10 & .77 & $.62-.87$ & 4.81 & .95 & $.87-.98$ & $-.33,-.05$ \\
\hline I31c & 4.00 & .75 & $.59-.85$ & 4.50 & .87 & $.77-.93$ & $-.29, .02$ \\
\hline $132 p$ & 4.20 & .80 & $.65-.89$ & 4.94 & .98 & $.91-.99$ & $-.33,-.07$ \\
\hline I32c & 4.20 & .80 & $.65-.89$ & 4.81 & .95 & $.87-.98$ & $-.30,-.03$ \\
\hline $133 p$ & 3.70 & .67 & $.52-.79$ & 4.93 & .98 & $.91-.99$ & $-.46,-.17$ \\
\hline $133 c$ & 3.80 & .70 & $.54-.81$ & 4.73 & .93 & $.83-.97$ & $-.39,-.08$ \\
\hline $134 p$ & 4.20 & .80 & $.65-.89$ & 4.88 & .97 & $.89-.99$ & $-.31,-.05$ \\
\hline I34c & 4.20 & .80 & $.65-.89$ & 4.50 & .87 & $.77-.93$ & $-.23, .06$ \\
\hline $135 p$ & 4.20 & .80 & $.65-.89$ & 4.88 & .97 & $.89-.99$ & $-.31,-.05$ \\
\hline $135 \mathrm{c}$ & 4.10 & .77 & $.62-.87$ & 4.50 & .87 & $.77-.93$ & $-.29, .04$ \\
\hline $136 p$ & 4.10 & .77 & $.62-.87$ & 4.56 & .89 & $.79-.94$ & $-.28, .02$ \\
\hline $136 c$ & 4.10 & .77 & $.62-.87$ & 4.06 & .76 & $.64-.85$ & $-.16, .17$ \\
\hline $137 p$ & 3.60 & .65 & $.49-.77$ & 4.94 & .98 & $.91-.99$ & $-.49,-.19$ \\
\hline I37c & 3.70 & .67 & $.52-.79$ & 4.31 & .82 & $.71-.90$ & $-.32, .01$ \\
\hline$A C$ & MD & VD & IC D 95\% & MI & VI & IC I 95\% & IC VD - VI \\
\hline $138 p$ & 4.20 & .80 & $.65-.89$ & 4.47 & .86 & $.75-.93$ & $-.23, .08$ \\
\hline $138 c$ & 4.00 & .75 & $.59-.85$ & 4.47 & .86 & $.75-.93$ & $-.28, .04$ \\
\hline $139 p$ & 4.30 & .82 & $.68-.91$ & 4.81 & .95 & $.87-.98$ & $-.27,-.01$ \\
\hline I39c & 4.30 & .82 & $.68-.91$ & 4.25 & .81 & $.70-.88$ & $-.15, .15$ \\
\hline $140 p$ & 4.20 & .80 & $.65-.89$ & 4.94 & .98 & $.91-.99$ & $-.33,-.07$ \\
\hline $140 c$ & 4.20 & .80 & $.65-.89$ & 4.75 & .93 & $.85-.97$ & $-.29,-.01$ \\
\hline $141 p$ & 4.20 & .80 & $.65-.89$ & 4.88 & .97 & $.89-.99$ & $-.31,-.05$ \\
\hline I41c & 4.20 & .80 & $.65-.89$ & 4.56 & .89 & $.79-.94$ & $-.25, .04$ \\
\hline $142 p$ & 4.20 & .80 & $.65-.89$ & 4.94 & .98 & $.91-.99$ & $-.33,-.07$ \\
\hline
\end{tabular}


Sánchez-Rosas, J., Dyzenchauz, M. \& Domínguez-Lara, S. / RACC, 2021, Vol. 13, №1, 59-72

\begin{tabular}{llllllll}
$\mathrm{I} 42 \mathrm{c}$ & 4.10 & .77 & $.62-.87$ & 4.69 & .92 & $.83-.96$ & $-.31,-.02$ \\
\hline $\mathrm{I} 43 \mathrm{p}$ & 4.20 & .80 & $.65-.89$ & 4.87 & .96 & $.88-.99$ & $-.31,-.04$ \\
$\mathrm{I} 43 \mathrm{c}$ & 4.20 & .80 & $.65-.89$ & 4.47 & .86 & $.75-.93$ & $-.23, .08$ \\
\hline $\mathrm{I} 44 \mathrm{p}$ & 4.20 & .80 & $.65-.89$ & 4.73 & .93 & $.83-.97$ & $-.29,-.01$ \\
$\mathrm{I} 44 \mathrm{c}$ & 4.20 & .80 & $.65-.89$ & 4.25 & .81 & $.70-.88$ & $-.18, .13$ \\
\hline $\mathrm{I} 45 \mathrm{p}$ & 4.20 & .80 & $.65-.89$ & 4.25 & .81 & $.70-.88$ & $-.18, .13$ \\
$\mathrm{I} 45 \mathrm{c}$ & 4.20 & .80 & $.65-.89$ & 4.56 & .89 & $.79-.94$ & $-.25, .04$ \\
\hline
\end{tabular}

Nota. I = ítem; MD = media docentes; VD = V de Aiken docentes; IC D = intervalos de confianza docentes; $\mathrm{MI}=$ media investigadores; $\mathrm{VI}=\mathrm{V}$ de Aiken investigadores; IC I = intervalos de confianza investigadores; ATD = Autoeficacia para influir en la toma de decisiones; $A E$ = Autoeficacia para la enseñanza; $A C E=$ Autoeficacia para la convivencia escolar; $\mathrm{APF}=$ Autoeficacia para el logro de la participación familiar; $\mathrm{APC}=$ Autoeficacia para el logro de la participación comunitaria; $A C$ = Autoeficacia para crear un clima positivo.

\section{Discusión}

La ACD se presenta como un constructo prometedor con gran influencia en numerosas variables estudiadas por la psicología educacional. Sin embargo, los instrumentos existentes para medirlas presentan limitaciones: exhiben una falta de congruencia con la teoría, fueron desarrollados en base a características de sus contextos nacionales, fueron construidos a partir de escalas de autoeficacia docente individual, y la redacción de los ítems se desvía de los parámetros sugeridos por Bandura (2006) para crear test de autoeficacia. Con el fin de indagar sobre este constructo desde una perspectiva cuantitativa, resulta indispensable contar con un instrumento capaz de medirlo. Es por esto que el presente trabajo se propuso la creación y la generación de evidencias de validez de contenido de un instrumento para evaluar la ACD. Estos objetivos fueron alcanzados satisfactoriamente, obteniendo una versión preliminarmente válida para ser utilizada en investigaciones posteriores con el fin de explorar nuevas evidencias psicométricas.

En el presente trabajo se emplearon dos muestras de validación: docentes e investigadores. Aunque el juicio de expertos acostumbra incluir investigadores expertos, se siguió la recomendación de Merino-Soto (2016) de incluir una muestra de la población destinataria del instrumento. Esto se debe a que, en ocasiones, la percepción de los investigadores sobre la claridad de los reactivos puede diferir en las poblaciones destinatarias, debido a factores como los conocimientos técnicos sobre la temática, la experiencia o las trayectorias profesionales.

Esto se comprueba en el presente trabajo: los resultados exhiben diferencias entre las valoraciones de las dos muestras. En general, los reactivos obtuvieron mejores puntajes (media, $\mathrm{V}$ de Aiken e IC) para la muestra de investigadores. Sin embargo, los ítems propuestos fueron bien valorados: la media de cada puntaje por parte de ambas muestras y tanto para la claridad como para la pertinencia resultó muy buena (> 3.50). Además, los comentarios de ambas muestras de jueces resultaron de utilidad para realizar algunas modificaciones puntuales, haciéndolos más breves y comprensibles. Por ejemplo, se eliminó la palabra podemos que se encontraba al principio de cada reactivo, agregándola en la consigna. Además, se reemplazaron algunos términos técnicos por nociones más familiares a la profesión docente y se modificó la nominación de algunas dimensiones. Se resalta la utilidad de contar con la participación de la muestra destinataria del instrumento (docentes), ya que normalmente se considera el juicio de investigadores como el que tiene la última palabra, pudiendo éstos llegar a dar opiniones sesgadas. Si bien los investigadores expertos pueden valorar a un ítem según su consistencia teórica y la corrección de su redacción, el mismo puede ser incomprensible o poco familiar para quienes finalmente responderán a la escala.

La propuesta de utilizar como muestra de jueces no sólo a investigadores sino a representantes de la población destinataria es un punto de partida para profundizar en las evidencias psicométricas de los instrumentos (Merino-Soto, 2018). Las diferencias halladas entre los puntajes de investigadores y docentes dan cuenta de esto, y llaman la atención sobre la importancia de considerar a la población objetivo como jueces que pueden otorgar información sobre la comprensibilidad de los ítems. Sin embargo, también evidencian que, debido a que la 
percepción de claridad entre docentes fue baja, es probable que los indicadores de confiabilidad se vean afectados sin un soporte durante el proceso de respuesta. Es por esto que se debería administrar la escala en grupos pequeños, contando con mayor orientación por parte del evaluador.

Los resultados obtenidos en este estudio son un primer paso en la validación de esta herramienta. En estudios futuros será necesario efectuar pruebas que evalúen la estructura interna del instrumento, indagando si la estructura dimensional propuesta se ajusta a la realidad. Por otra parte, podría brindarse evidencias de validez convergente 0 divergente al relacionar las puntuaciones de esta escala con las una de autoeficacia docente individual validada al español (Domínguez-Lara, Fernández-Arata, Merino-Soto, Navarro-Loli, \& Calderón-De la Cruz, 2019). Finalmente, podrían efectuarse estudios de validez test-criterio, verificando la relación de las dimensiones con otros constructos que pueden considerarse relacionados teóricamente, como la satisfacción laboral docente, el síndrome de burnout (Buonomo, Fiorilli, \& Benevene, 2020; Malinen \& Savolainen, 2016), el sentido de pertenencia a la institución y el compromiso docente (Skaalvik, E. \& Skaalvik, 2019).

\section{Conclusiones}

El presente estudio aportó evidencias de validez de contenido para la Escala de ACD. Los ítems demostraron ser comprensibles y pertinentes a las dimensiones propuestas para el constructo. Además, se encontraron diferencias entre las valoraciones de investigadores y docentes que resultaron útiles para guiar la administración del instrumento. Los comentarios realizados por ambas muestras también sirvieron para efectuar algunas modificaciones en los ítems, haciéndolos más claros y breves.

\section{Referencias}

Adams, C. M., \& Forsyth, P. B. (2006). Proximate sources of collective teacher efficacy. Journal of Educational Administration, 44(6), 625-642. doi: 10.1108/09578230610704828

Bandura, A. (2006). Guide for constructing self-efficacy scales. En F. Pajares, \& T. Urdan (Eds.), SelfEfficacy Beliefs of Adolescents (pp. 307-337). Greenwich, CT: Information Age Publishing.

Buonomo, I., Fiorilli, C., \& Benevene, P. (2020).
Unravelling Teacher Job Satisfaction: The Contribution of Collective Efficacy and Emotions Towards Professional Role. International Journal of Environmental Research and Public Health, 17(3), 736. doi:10.3390/ijerph17030736

Cantrell, S. C., \& Callaway, P. (2008). High and low implementers of content literacy instruction: Portraits of teacher efficacy. Teaching and Teacher Education, 24(7), 1739-1750. doi: 10.1016/j.tate.2008.02.020

Centro de Implementación de Políticas Públicas para la Equidad y el Crecimiento [CIPPEC] (2017). Aprender 2016. Características y Voces de los Docentes. Secretaría de Evaluación Educativa, Ministerio de Educación. Recuperado de: https://www.cippec.org/wpcontent/uploads/2018/03/caracteristicas_y_voces_ de_los_docentes_web_a4_simple.pdf

Cooper, J. D. (2010). Collective Efficacy, Organizational Citizenship Behavior and School Effectiveness in Alabama Public High Schools (Tesis doctoral). Department of Educational Leadership, Policy and Technology Studies in the Graduate School of The University of Alabama, Tuscaloosa, Alabama. Recuperado de: https://ir.ua.edu/bitstream/handle/123456789/922/fil e_1.pdf?sequence $=1$

Cuenca, R., Fabara Garzón, E., Kohen, J., Parra Garrido, M., Rodríguez Guzmán, L., \& Tomasina, F. (2005). Condiciones de salud y trabajo docente. Chile: Oficina Regional de Educación de la UNESCO para América Latina y el Caribe.

Domínguez-Lara, S. (2017). Construcción de una escala de autoeficacia para la investigación: primeras evidencias de validez. Revista Digital de Investigación en Docencia Universitaria, 11(2), 308-322. doi: 10.19083/ridu.11.514

Domínguez-Lara, S., Fernández-Arata, M., MerinoSoto, C., Navarro-Loli, J., \& Calderón-De la Cruz, G. (2019). Escala de Autoeficacia Docente: análisis estructural e invarianza de medición en docentes peruanos de escuelas públicas. Revista Argentina de Ciencias del Comportamiento, 11(3), 61-72.

Dussault, M., Payette, D., \& Leroux, M. (2008). Principals' Transformational Leadership and Teachers' Collective Efficacy. Psychological Reports, 102(2), 401-410. doi: 10.2466/pro.102.2.401-410

Esquivel, S., \& Sánchez-Rosas, J. (2018). Calidad Instruccional Docente, Emociones, Valor de la Tarea, Autoeficacia y Atención en Clases de Nivel Universitario. Anuario de Investigaciones de la Facultad de Psicología, 3(3), 31-39.

Fives, H., \& Looney, L. (2009). College instructors' sense of teaching and collective efficacy. International Journal of Teaching and Learning in Higher Education, 20(2), 182-191. 
Gibson, S., \& Dembo, M. H. (1984). Teacher efficacy: A construct validation. Journal of Educational Psychology, 76(4), 569-582. doi:10.1037/00220663.76.4.569

Goddard, R. (2002). A theoretical and empirical analysis of the measurement of collective efficacy: The development of a short form. Educational and Psychological Measurement, 62(1), 97-110. doi: 10.1177/0013164402062001007

Goddard, R. D., Hoy, W. K., \& Woolfolk Hoy, A. (2000). Collective Teacher Efficacy: Its Meaning, Measure, and Impact on Student Achievement. American Educational Research Journal, 37(2), 479-507. doi: 10.3102/00028312037002479

Gregory, A., Skiba, R. J., \& Noguera, P. A. (2010). The Achievement Gap and the Discipline Gap: Two Sides of the Same Coin? Educational Researcher, 39(1), 59-68. doi: 10.3102/0013189X09357621

Jean Eells, R. (2011). Meta-Analysis of the Relationship Between Collective Teacher Efficacy and Student Achievement (Tesis Doctoral). Loyola University, Chicago. Recuperado de: https://ecommons.luc.edu/luc_diss/133

Kim, S. W., \& Hill, N. E. (2015). Including fathers in the picture: A meta-analysis of parental involvement and students' academic achievement. Journal of Educational Psychology, 107(4), 919-934. doi: 10.1037/edu0000023

Kirby, M. M., \& DiPaola, M. F. (2011). Academic optimism and community engagement in urban schools. Journal of Educational Administration, 49(5), 542-562. doi: 10.1109/09578231111159539

Klassen, R. M. (2010). Teacher stress: The mediating role of collective efficacy beliefs. Journal of Educational Research, 103(5), 342-350. doi: 10.1080/00220670903383069

Klassen, R. M., Tze, V. M. C., Betts, S. M., \& Gordon, K. A. (2011). Teacher Efficacy Research 19982009: Signs of Progress or Unfulfilled Promise? Educational Psychology Review, 23(1), 21-43. doi: 10.1007/s10648-010-9141-8

Klassen, R. M., Usher, E. L., \& Bong, M. (2010). Teachers' collective efficacy, job satisfaction, and job stress in cross-cultural context. Journal of Experimental Education, 78(4), 464-486. doi: 10.1080/00220970903292975

Koth, C. W., Bradshaw, C. P., \& Leaf, P. J. (2008). A Multilevel Study of Predictors of Student Perceptions of School Climate: The Effect of Classroom-Level Factors. Journal of Educational Psychology, 100(1), 96-104. doi: 10.1037/00220663.100.1.96

Kurz, T. B., \& Knight, S. L. (2004). An exploration of the relationship among teacher efficacy, collective teacher efficacy, and goal consensus. Learning Environments Research, 7(2), 111-128. doi: 10.1023/B:LERI.0000037198.37750.0e
Lee, J. C. K., Zhang, Z., \& Yin, H. (2011). A multilevel analysis of the impact of a professional learning community, faculty trust in colleagues and collective efficacy on teacher commitment to students. Teaching and Teacher Education, 27(5), 820-830. doi: 10.1016/j.tate.2011.01.006

Leithwood, K., Sun, J., \& Schumacker, R. (2019). How School Leadership Influences Student Learning: A Test of "The Four Paths Model." Educational Administration Quarterly, 56(4), 570-599. doi: 10.1177/0013161X19878772

Lev, S., \& Koslowsky, M. (2009). Moderating the collective and self-efficacy relationship. Journal of Educational Administration, 47(4), 452-462. doi: 10.1108/09578230910967437

Lim, S., \& Eo, S. (2014). The mediating roles of collective teacher efficacy in the relations of teachers' perceptions of school organizational climate to their burnout. Teaching and Teacher Education, 44, 138-147. doi: 10.1016/j.tate.2014.08.007

Ma, L., Resendes, M., Scardamalia, M., \& Dobbie, K. (Mayo de 2019). The knowledge building network pilot project: An exploration of emergent designs to enhance collective teacher efficacy. Conferencia presentada en Computer-Supported Collaborative Learning Conference (CSCL), Ontario, Canadá.

Malinen, O. P., \& Savolainen, H. (2016). The effect of perceived school climate and teacher efficacy in behavior management on job satisfaction and burnout: A longitudinal study. Teaching and Teacher Education, 60, 144-152. doi: 10.1016/j.tate.2016.08.012

Mendoza, C. D. (2012). Parental Involvement and Student Motivation: A Quantitative Study of the Relationship between Student Goal Orientation and Student Perceptions of Parental Involvement among 5th Grade Students (Tesis doctoral). University of Southern California, California, Estados Unidos. Recuperado de: http://digitallibrary.usc.edu/cdm/ref/collection/p1579 9coll3/id/101550

Merino-Soto, C. (2016). Percepción de la claridad de los ítems: Comparación del juicio de estudiantes y jueces expertos. Revista Latinoamericana de Ciencias Sociales, Niñez y Juventud, 14(2), 14691477. doi: 10.11600/1692715x.14239120615

Merino-Soto, C. (2018). Confidence interval for difference between coefficients of content validity (Aiken's V): A SPSS syntax. Anales de Psicología, 34(3), 587-590. doi: 10.6018/analesps.34.3.283481

Merino-Soto, C., \& Livia Segovia, J. (2009). Intervalos de confianza asimétricos para el índice la validez de contenido: un programa Visual Basic para la $\mathrm{V}$ de Aiken. Anales de Psicología, 25(1), 159-161.

Michael, S., Dittus, P., \& Epstein, J. (2007). Family and 
community involvement in schools: Results from the school health policies and programs study 2006. Journal of School Health, 77(8), 567-579. doi: 10.1111/j.1746-1561.2007.00236.x

Montero, I., \& León, O. G. (2007). Guía para nombrar los estudios de investigación en Psicología. International Journal of Clinical and Health Psychology, 7(3), 847-862.

Muñiz, J., \& Fonseca-Pedrero, E. (2019). Diez pasos para la construcción de un test. Psicothema, 31(1), 7-16. doi: 10.7334/psicothema2018.291

Olivier, D. F., \& Hipp, K. K. (2019). Leadership Capacity and Collective Efficacy: Interacting to Sustain Student Learning in a Professional Learning Community. Journal of School Leadership, 16(5), 505-519. doi: 10.1177/105268460601600504

Rodríguez, S., Núñez, J., Valle, A., Blas, R., \& Rosario, P. (2009). Auto-eficacia Docente, Motivación del Profesor y Estrategias de Enseñanza. Escritos de Psicología, 3(1), 1-7.

Sánchez-Rosas, J., \& Esquivel, S. (2016). Instructional Teaching Quality, Task Value, Self-Efficacy, and Boredom: A Model of Attention in Class. Revista de Psicología, 25(2), 1-20. doi: 10.5354/07190581.2016 .44966

Sánchez-Rosas, J., Takaya, P. B., \& Molinari, A. V. (2016a). Attention in Class: Predictive Role of Teacher Behavior, Task Value, Self-Efficacy, Enjoyment and Shame. Psiencia, Latin American Journal of Psychological Science, 8(3), 1-26. doi: 10.5872/psiencia/8.3.22

Sánchez-Rosas, J., Takaya, P. B., \& Molinari, A. V. (2016b). The Role of Teacher Behavior, Motivation and Emotion in Predicting Academic Social Participation in Class. Pensando Psicología, 12(19), 39-53. doi: 10.16925/pe.v12i19.1327

Skaalvik, E., \& Skaalvik, S. (2007). Dimensions of teacher self-efficacy and relations with strain factors, perceived collective teacher efficacy, and teacher burnout. Journal of Educational Psychology, 99(3), 611-625. doi: 10.1037/00220663.99.3.611

Skaalvik, E., \& Skaalvik, S. (2016). Teacher Stress and Teacher Self-Efficacy as Predictors of Engagement, Emotional Exhaustion, and Motivation to Leave the Teaching Profession. Creative Education, 7(13), 1785-1799. doi: 10.4236/ce.2016.713182

Skaalvik, E., \& Skaalvik, S. (2019). Teacher SelfEfficacy and Collective Teacher Efficacy: Relations with Perceived Job Resources and Job Demands, Feeling of Belonging, and Teacher Engagement. Creative Education, 10(7), 1400-1424. doi: 10.4236/ce.2019.107104

Somech, A. (2010). Participative decision making in schools: A mediating-moderating analytical framework for understanding school and teacher outcomes. Educational Administration Quarterly, 46(2), 174-209. doi: 10.1177/1094670510361745

Thapa, A., Cohen, J., Guffey, S., \& HigginsD'Alessandro, A. (2013). A Review of School Climate Research. Review of Educational Research, 83(3), 357-385. doi: 10.1177/1094670510361745

Tschannen-Moran, M., \& Barr, M. (2004). Fostering Student Learning: The Relationship of Collective Teacher Efficacy and Student Achievement. Leadership and Policy in Schools, 3(3), 189-209. doi:10.1080/15700760490503706

Tschannen-Moran, M., \& Woolfolk Hoy, A. (2001). Teacher efficacy: Capturing an elusive construct. Teaching and Teacher Education, 17(7), 783-805. doi: 10.1016/s0742-051x(01)00036-1

Vera-Villaroel, P., \& Oblitas, L. A. (2005). Manual de escalas $y$ cuestionarios iberoamericanos en psicología clínica y de la salud. Bogotá: Psicom Editores.

Wagner, J. A. (1994). Participation's effects on performance and satisfaction: a reconsideration of research evidence. Academy of Management Review, 19(2), 312-330. doi:1 0.2307/258707 


\section{Anexo}

Utilizá la siguiente escala para puntuar las afirmaciones en la columna derecha de la tabla
1
2
3
4
5
6
7
8
9

nada

Totalmente

seguro/a seguro/a

de que

podamos

hacerlo

de que

\section{Los y las docentes de esta escuela podemos...}

1. Organizarnos para ser escuchados al tomar decisiones en la escuela

2. Llegar a consensos para expresar nuestra opinión sobre cuestiones importantes para la escuela

3. Decidir de manera democrática aspectos que definen la identidad de la escuela

4. Reunirnos sistemáticamente para debatir sobre cuestiones de la cotidianidad escolar

5. Establecer vías de comunicación efectivas para incluir a todo el personal en la toma de decisiones

6. Ponernos de acuerdo sobre cómo comunicarles nuestra postura a los directivos de la institución

7. Organizarnos para demandar una intervención ante una situación problemática

8. Transmitir propuestas a las autoridades, referidas a la organización de la institución

9. Compartir estrategias efectivas para mejorar el desempeño de los estudiantes con dificultades de aprendizaje

10. Desarrollar en conjunto recursos didácticos para que los estudiantes trabajen en grupo

11. Compartir entre nosotros metodologías innovadoras para promover aprendizajes significativos

12. Desarrollar conjuntamente propuestas innovadoras que despierten en los estudiantes la 
Sánchez-Rosas, J., Dyzenchauz, M. \& Domínguez-Lara, S. / RACC, 2021, Vol. 13, №1, 59-72

\begin{tabular}{|l|l|}
\hline curiosidad y la creatividad & \\
\hline $\begin{array}{l}\text { 13. Desarrollar en conjunto estrategias para que los estudiantes se comprometan con su } \\
\text { aprendizaje }\end{array}$ & \\
\hline $\begin{array}{l}\text { 14. Trabajar un mismo tema de manera transversal en varias asignaturas para que los } \\
\text { estudiantes logren una mejor comprensión }\end{array}$ & \\
\hline $\begin{array}{l}\text { 15. Desarrollar en conjunto instancias lúdicas para motivar a los estudiantes a incorporar } \\
\text { contenidos de manera creativa }\end{array}$ & \\
\hline 16. Promover en conjunto que nuestros estudiantes adquieran técnicas y hábitos de estudio & \\
\hline 17. Contribuir entre todos a que los estudiantes respeten las reglas escolares & \\
\hline 18. Establecer acuerdos de convivencia junto con los estudiantes y el resto del personal & \\
\hline 19. Consensuar criterios que definan los comportamientos que se esperan de los estudiantes & \\
\hline 20. Consensuar las medidas que tomaremos ante conductas disruptivas de los estudiantes & \\
\hline 21. Intercambiar estrategias que hayan resultado exitosas para regular el comportamiento & \\
\hline $\begin{array}{l}\text { 22. Comunicar a los estudiantes de manera coherente el comportamiento que esperamos de } \\
\text { ellos en la escuela }\end{array}$ & \\
\hline 23. Desarrollar estrategias en conjunto para que los estudiantes se autoevalúen y aprendan & \\
a solucionar conflictos & \\
\hline 24. Trabajar en conjunto para que las familias acompañen a sus hijos en las tareas escolares & \\
\hline 25. Organizar actividades para que las familias se sientan parte de la institución & \\
\hline 26. Organizar reuniones para conversar con las familias sobre problemáticas escolares & \\
\hline 27. Establecer vías de comunicación que faciliten la participación de la familia & \\
\hline 28. Mostrar disponibilidad como equipo docente para que las familias cuenten con nosotros & \\
\hline 29. Organizar reuniones para que las familias participen de las decisiones institucionales & \\
\hline 30. Proponer en conjunto estrategias para fomentar junto con la familia hábitos de estudio & \\
\hline 31. Diseñar proyectos que involucren la participación de organizaciones comunitarias & \\
\hline $\begin{array}{l}\text { 32. Organizar jornadas con expertos de nuestra comunidad para conversar sobre temáticas } \\
\text { que nos interesen }\end{array}$ & \\
\hline 33. Crear redes de colaboración mutua con organismos de la comunidad & \\
\hline
\end{tabular}


Sánchez-Rosas, J., Dyzenchauz, M. \& Domínguez-Lara, S. / RACC, 2021, Vol. 13, №1, 59-72

34. Organizar actividades recreativas donde participen personas externas a la institución

35. Organizar entre todos actividades educativas donde participen personas externas a la institución

36. Darnos a conocer como institución ante la comunidad

37. Participar como institución en eventos extraescolares

38. Entablar relaciones positivas para hacer de la escuela un lugar confiable

39. Fomentar entre todos que los estudiantes confíen en nosotros

40. Crear espacios de reflexión grupal para promover relaciones empáticas

41. Desarrollar actividades que refuercen el sentido de pertenencia a la institución en los estudiantes

42. Garantizar entre todos la inclusión y el respeto por la diversidad

43. Ayudarnos entre nosotros cuando surge alguna dificultad

44. Realizar actividades para encontrarnos de manera distendida entre todos los agentes de la escuela

45. Reflexionar sobre las problemáticas escolares y abordarlas en conjunto 